\title{
Bit Synchronization of Data Transmission Signal Based On Instantaneous Frequency Measurement
}

\author{
Gang Fu, Jian Chen, Yue Feng
}

China Satellite Maritime Tracking and Control Department, Jiangyin, 214431, China

\begin{abstract}
This article discusses the software demodulation bit synchronization system implementation. According to the characteristics and DSTFT DQPSK signal demodulation characteristics studied a bit synchronization algorithms: synchronization based on the instantaneous frequency measurement position, the signal instantaneous frequency measurement by looking symbols starting position.
\end{abstract}

Keywords: PCM-QPSK; Instantaneous frequency measurement; Ssynchronization.

\section{Introduction}

Coding include telemetry synchronization bit synchronization, word synchronization, frame synchronization and the sub frame synchronization. Bit synchronization is the basis for proper communication, and only after obtaining the correct bit synchronization word to achieve synchronization, frame synchronization and the sub frame synchronization. Word for word synchronizing data divided by the number of code bits composed. PCM telemetry word usually fixed, with the rear frame synchronization and bit synchronization, word synchronization can be easily achieved without special word synchronization signal.

\section{Frequency measurement based on phase estimation method}

For the signal sequence:

$S_{i}=A \sin \left(\omega T_{S} i+\varphi\right)$

Observed wherein m continuous sampling points are:

$S_{i+k}=A \sin \left[\omega T_{s}(i+k)+\varphi\right], \quad k=0,1, \cdots, m-1$

And the middle point of this section of the signal corresponding to the phase as follows:

$\varphi_{i}=\varphi+\omega T_{s}\left(i+\frac{m-1}{2}\right)$

The equation (1) becomes:

$$
\begin{aligned}
S_{i+k} & =A \sin \left[\varphi_{i}+\omega T_{s}\left(k-\frac{m-1}{2}\right)\right] \\
& =\cos \left[\omega T_{s}\left(k-\frac{m-1}{2}\right)\right] \cdot A \sin \left(\varphi_{i}\right)+\sin \left[\omega T_{s}\left(k-\frac{m-1}{2}\right)\right] \cdot A \cos \left(\varphi_{i}\right)
\end{aligned}
$$

Assume that the matrix $\mathrm{S}$ is composed of $\mathrm{m}$ sample points, CS is the matrix of coefficients $\cos \left[\omega T_{s}\left(k-\frac{m-1}{2}\right)\right]$ and $\sin \left[\omega T_{s}\left(k-\frac{m-1}{2}\right)\right]$ form, PH to $A \sin \left(\varphi_{i}\right)$ and $A \cos \left(\varphi_{i}\right)$ matrix composition. Thus equation (3) may be expressed as:

$S=C S \cdot P H$

Depending $\mathrm{PH}$ is unknown, solving the equation by the least squares, generalized inverse is expressed as:

$P H=C S^{-1} \cdot S$

According to $\mathrm{PH}$ clues can be obtained:

$$
\varphi_{i}=\arctan \left[\frac{\sum_{k=0}^{m-1} \cos \left[\omega T_{s}\left(k-\frac{m-1}{2}\right)\right] \cdot S_{i+k}}{\sum_{k=0}^{m-1} \sin \left[\omega T_{s}\left(k-\frac{m-1}{2}\right)\right] \cdot S_{i+k}} \cdot \frac{1-\frac{\sin \left(m \omega T_{s}\right)}{m \sin \left(\omega T_{s}\right)}}{1+\frac{\sin \left(m \omega T_{s}\right)}{m \sin \left(\omega T_{s}\right)}}\right]
$$


After the phase of the signal by the formula (6) is obtained, it is possible (3) with the principle of least squares regression to strike again with the frequency of the signal according to the formula:

$$
f=12 \sum_{i=1}^{n}\left[\frac{\varphi_{i}}{2 \pi}\left(i-\frac{n+1}{2}\right)\right] \cdot \frac{1}{n\left(n^{2}-1\right)} \cdot f_{s}
$$

Where, $\mathrm{n}$ is the number of points estimated phase when the frequency of use.

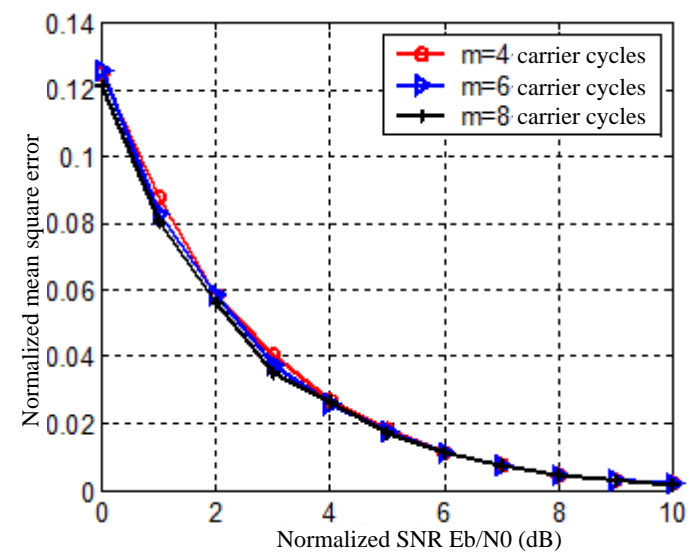

(a). Frequency measurement method estimated frequency error

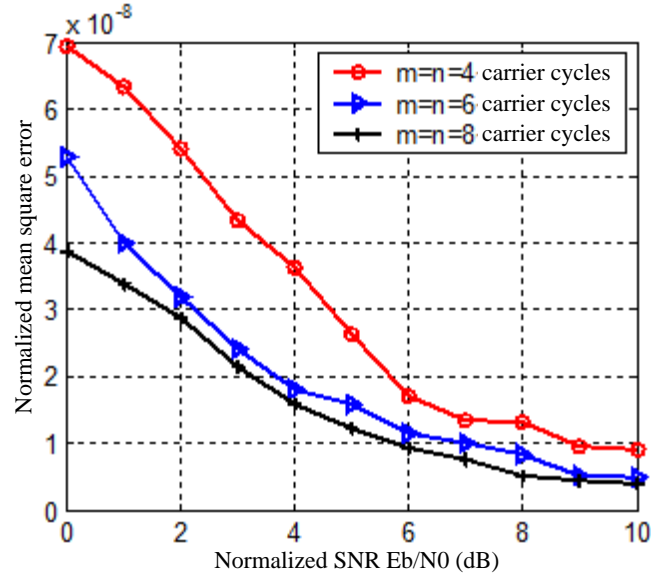

(b). Phase estimation error frequency measurement method

Figure 1. Frequency estimate normalized mean square error

Figure 1 shows the frequency and phase estimation method projection method for a single-carrier frequency error estimation signal, carrier signal parameter is $2.1 \mathrm{MHz}$, and the sampling rate of $16 \mathrm{Msps}$. Frequency estimate normalized mean square error is defined as:

$$
E\left(|\varepsilon|^{2}\right)=E\left(\left|\frac{f-\hat{f}}{f}\right|^{2}\right)
$$

Both the frequency is shown in Figure 1. Comparative estimation error can be seen in poor frequency reckoning frequency measurement accuracy. The actual demodulation using phase estimation method DQPSK signal instantaneous frequency measurement and positioning the starting position of the symbol, the entire process is shown in Figure 2.

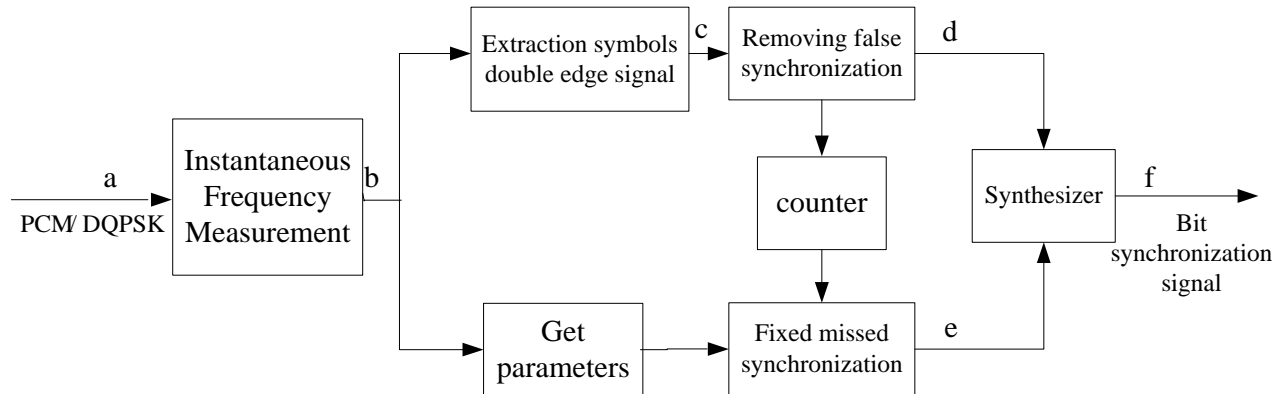

Figure 2. Instantaneous frequency measurement bit synchronization 
Waveform diagram is given in each processing figure 2, corresponding to Figure 3.

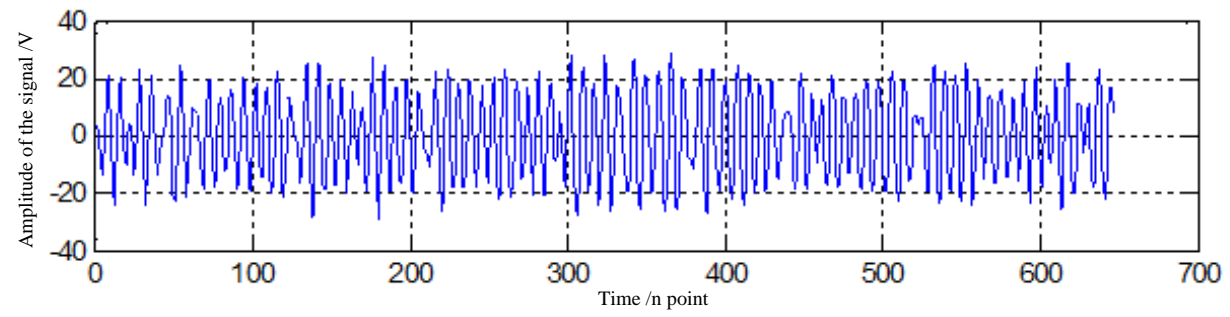

(a). QPSK signal in time domain

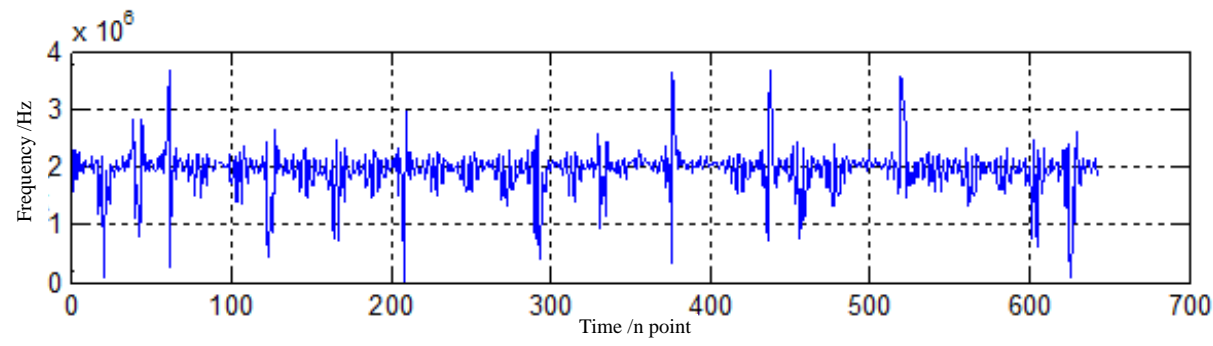

(b). Results of instantaneous frequency measurement

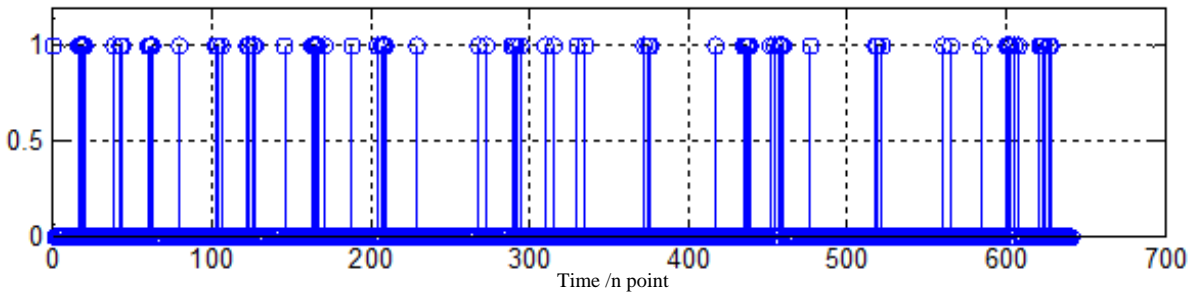

(c). Double edge signal obtained by frequency judgment

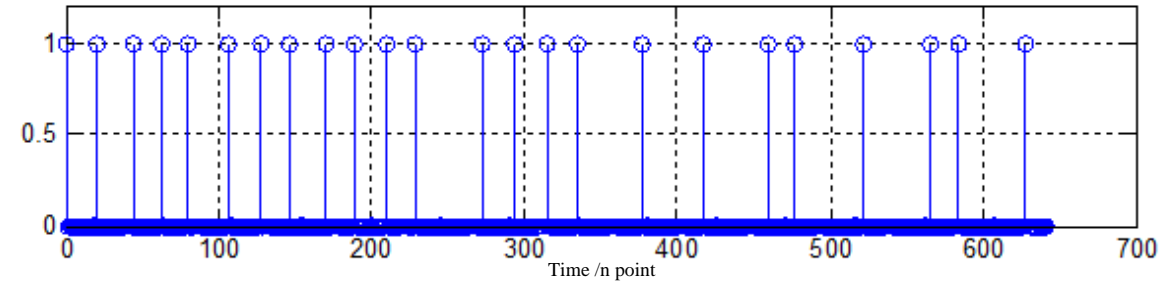

(d). Remove the false synchronization

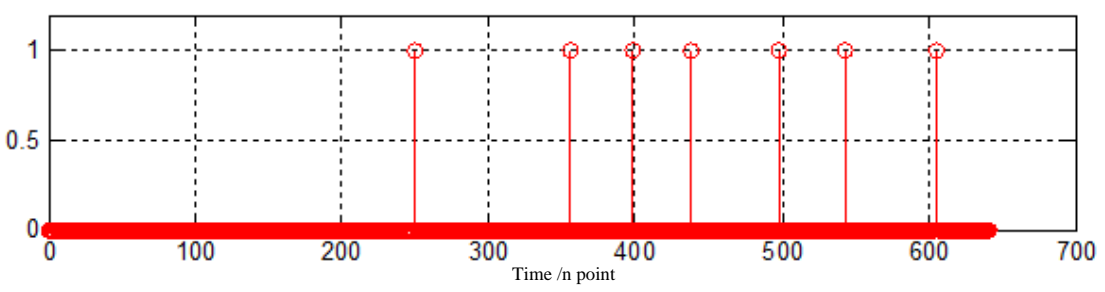

(e). Added missed synchronization

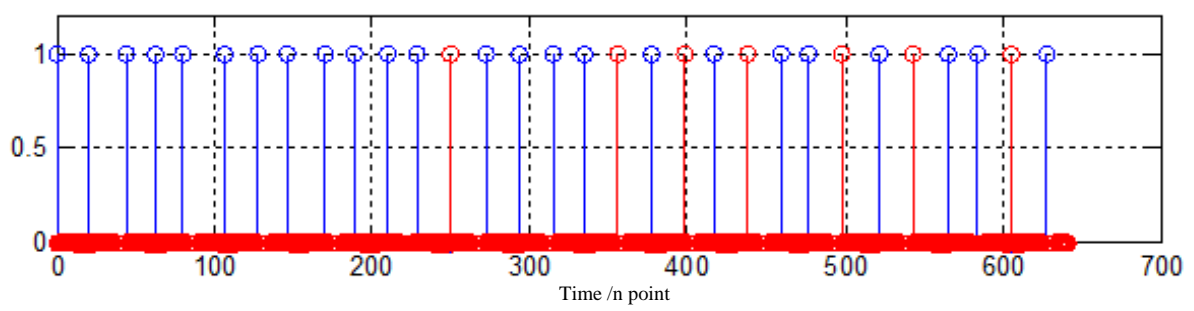

(f). Bit synchronization signal (symbol start point)

Figure 3. Synchronization waveform based on the instantaneous frequency measurement bit

Through computer simulation, it will be used for differential IF demodulation bit synchronization method based on instantaneous frequency measurement. IF differential demodulator into a baseband 
signal input judgment, the best judgment and bit synchronization timing waveform obtained demonstrated in Figure 4.
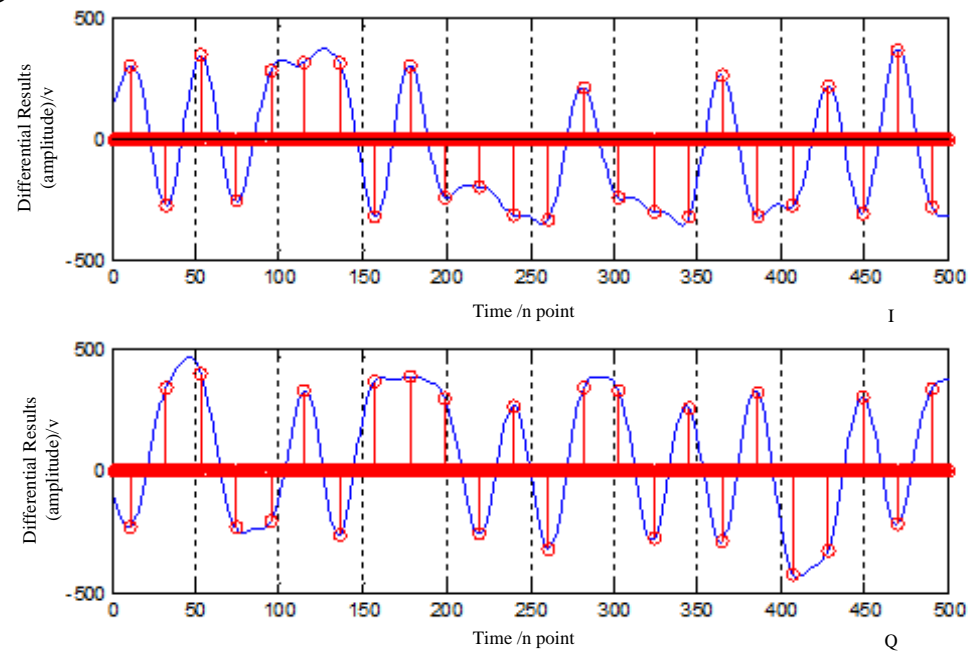

Figure 4. Judgment before the baseband waveform and best decision time

As can be seen from Figure 4, the use of instantaneous frequency measurement method can accurately locate the best judgment of each moment; it proves the correctness and feasibility of such a bit synchronization method.

\section{Summary}

This article discusses the software demodulation bit synchronization system implementation. According to the characteristics and DSTFT DQPSK signal demodulation characteristics studied a bit synchronization algorithms: synchronization based on the instantaneous frequency measurement position, the signal instantaneous frequency measurement by looking symbols starting position.

\section{References}

[1]. L.Erup, F,M, Gardner, interpolation in digital modems-part II . Implementation and performance, IEEE. Trans. Com, Jun.2013, 41(6):998-1008.

[2]. Chuang J.C, Sollenberger N.R, Burst coherent demodulation with combined symbol timing frequency offset estimation, and diversity selection. IEEE, Trans.Com, 2011, 39(7):1157-1164.

[3]. Sollemberger N.R, Chuang J.C, Low Overhead symbol timing and carrier recovery for TDMA portable radia systems. IEE, Trans.Com, 2010, 38(10):1886-1892.

[4]. G.Ascheid, M.oerder, H.meyr, An All Digital Receiver Architecture for Bandwidth Efficient Transmussion at high Rates, IEEE, Trans. Com, Aug.2009, 37(8):804-813.

[5]. D.Divsalar and M. K.Simon. Multiple-symbol differential detection of MPSK [J]. IEEE Trans, Communm, 2010, 38(3):300-308.

[6]. H.Leib, S.Pasupathy. The phase of a vector perturbed by Gaussian noise and differentially coherent receivers [J]. IEEE Trans. Inform, Theory, 2008, 34(6):1491-1501. 